\title{
A Preliminary Study on the Statistical Characters of the Atmospheric Turbulence
}

\author{
By M. Hayashi* \\ Institute of Oceanography, University of British Columbia, Vancouver, B.C., Canada. \\ (Manuscript received 12 Feburary 1974, in revised form 2 August 1974)
}

\begin{abstract}
Some statistical characters of the turbulent fluctuation in the atmospheric boundary layer are studied experimentally in a stability and frequency domain. The density functions of longitudinal and vertical velocity and temperature fluctuations are expanded in the Gram-Charlier series. The stability dependency of the expansion coefficient is discussed in relation to feature of turbulence. A running mean filter is used to separate the fluctuation into convective and inertial range.
\end{abstract}

\section{Introduction.}

The turbulence in wind tunnel or atmosphere is an example of random processes. Normality is a good approximation for the probability distribution of turbulent velocity fluctuation in wind tunnel (Batchelor, 1953). This feature is a little different for the turbulence in atmospheric boundary layer. Under unstable condition, a few rapidly rising current of hot air is surrounded by large area of slowly subsiding motion of cold air (Priestley, 1959). The distributions of vertical velocity and temperature fluctuations may not be normal as in wind tunnel or neutral stability. This paper studies some statistical characters of the turbulent fluctuation in the atmospheric boundary layer experimentally in stability and frequency domain.

If $x$ is a random variable and the quantity

$$
\mu_{n}=\int_{-\infty}^{\infty} x^{n} B(x) d x
$$

exists, $\mu_{n}$ is called the $n$-th moment of the variable $x$. $B(x)$ is the density function of $x$. The moments give information about the shape of the density function. The even central moment, moment about the mean, gives information about width of the density function and the odd lack of symmetry. The second central moment is called the variance or the square of the familiar standard deviation $\sigma$.

* Present affiliation is National Institute for Pollution and Resources, Tokyo.
When a density function is close to a normal, the Gram-Charlier representation may be convenient to show a correction to a normal distribution (Kendall and Stuart, 1969; Lumley, 1970). The deviation from a normal is given by the coefficients of the series expansion. For simplisity we take those moments about the mean and the signal in standard measure. Then the expansion can be written as

$$
B(x)=\alpha(x)\left\{1+\frac{1}{6} \mu_{3} H_{3}+\frac{1}{24}\left(\mu_{4}-3\right) H_{4}+\cdots\right\}
$$

where

$$
\alpha(x)=\frac{1}{\sqrt{(2 \pi)}} \exp \left(-\frac{1}{2} x^{2}\right)
$$

The functions $H_{n}$ are called the Hermit polynomials. The first few are given by

$$
\begin{aligned}
& H_{3}=x^{3}-3 x \\
& H_{4}=x^{4}-6 x^{2}+3
\end{aligned}
$$

The expansion coefficients $\mu_{n}$ are given by eq. (1). $\mu_{3}$ and $\mu_{4}-3$ are called skewness and kurtosis respectively and written as $S$ and $K$ with suffix of component. The skewness is a direct indication of the asymmetry of a density function and sometimes called coefficient of asymmetry. Skewness and kurtosis are zero when a density function is normal and not zero for non-normal density function.

According to the Monin-Obukhov's similarity theory, the standard deviation nondimensionalised 
by friction velocity $u^{*}$ or scaling tempetature $T^{*}$ is a universal function of the stability parameter, $z / L$, where $z$ is the height of the observation and $L$ the Monin-Obukhov length. The statistically higher order terms, skewness and kurtosis, are also expected to be universal functions of the stability parameter $z / L$. Because, the shape of the density function depends on the feature of turbulence.

In the exactly similar way, the moment of joint density function may be defined. The first moment is called the correlation or the covariance. The correlation coefficient $\rho_{i j}$ is the correlation nondimensionalised by the standard deviation of each variable component,

$$
\rho_{i j}=\frac{1}{\sigma_{i} \sigma_{j}} \iint_{-\infty}^{\infty} x_{i} x_{j} B\left(x_{i}, x_{j}\right) d x_{i} d x_{j}
$$

where $B\left(x_{i}, x_{j}\right)$ is the joint density function of variables $x_{i}$ and $x_{j}$. The correlation coefficient is also discussed in stability domain. The graphic method is used to represent the observed density function as Lumley and Tennekes (1970) employed to explaine the characters of turbulence schematically. Holland $(1967,1968)$ showed some illustrative examples of joint density functions. A typical example of the observed joint density function is shown here in frequency domain.

\section{Experimental arrangement and data processing.}

The data were collected from the International Field Experiment conducted at Tsmilyansk, USSR (Tsvang and Miyake, 1970). A group of the Institute of Oceanography at University of British Columbia measured the fluctuations of longitudinal and vertical velocities, $u$ and $w$, and temperature, $T$, by means of an acoustic anemometer-thermometer. The instrument was mounted on a tower at a height of 4 or $5 \mathrm{~m}$. 20 runs are chosen for analysis. Each run is consist of 20-30 minutes duration. Throughout these runs the wind speed was $5-8 \mathrm{~m} / \mathrm{sec}$. The stability was near neutral or weak unstable in most cases. The stability is specified by $z / L$. The Monin-Obukhov length $L$ is given by

$$
L=-\frac{u^{* 2} T}{k^{2} g T^{*}}
$$

and calculated by the direct measurements of $u^{*}\left(=\sqrt{-\overline{u^{\prime} w^{\prime}}}\right)$ and $T^{*}\left(=\overline{T^{\prime} w^{\prime}} / k u^{*}\right)$ using the eddy correlation method. All quantities have usual meanings.
Each signal is digitized at 50 samples per second. To avoid an error caused by quantization, the spread of signal is checked so that the full range of the data should be exceed 300 digital units (Bendat and Piersol, 1966). In this case the r.m.s. noise-to-signal ratio is estimated about 0.001. 15 unstable cases are summarised into three groups according to the stability and the averaged values are designated with the standard error. For stable case each run is plotted on graphs.

The expansion coefficient of the Gram-Charlier series, skewness and kurtosis, are checked by two different methods. One is that the density function $B(x)$ is obtained first as a histgram on 1023 class intervals and the moments are calculated by eq. (1). Another is that the $n$-th moment $\mu_{n}$ is calculated by

$$
\mu_{n}=\frac{1}{N} \sum_{i=1}^{N} x_{i}{ }^{\prime n}
$$

where $x_{i}{ }^{\prime}$ is in standard measure;

$$
x_{i}{ }^{\prime}=\left(x_{i}-\mu_{1}\right) / \sqrt{\mu_{2}}
$$

$x_{i}$ is raw data, $\mu_{1}$ the mean value and $\mu_{2}$ variance.
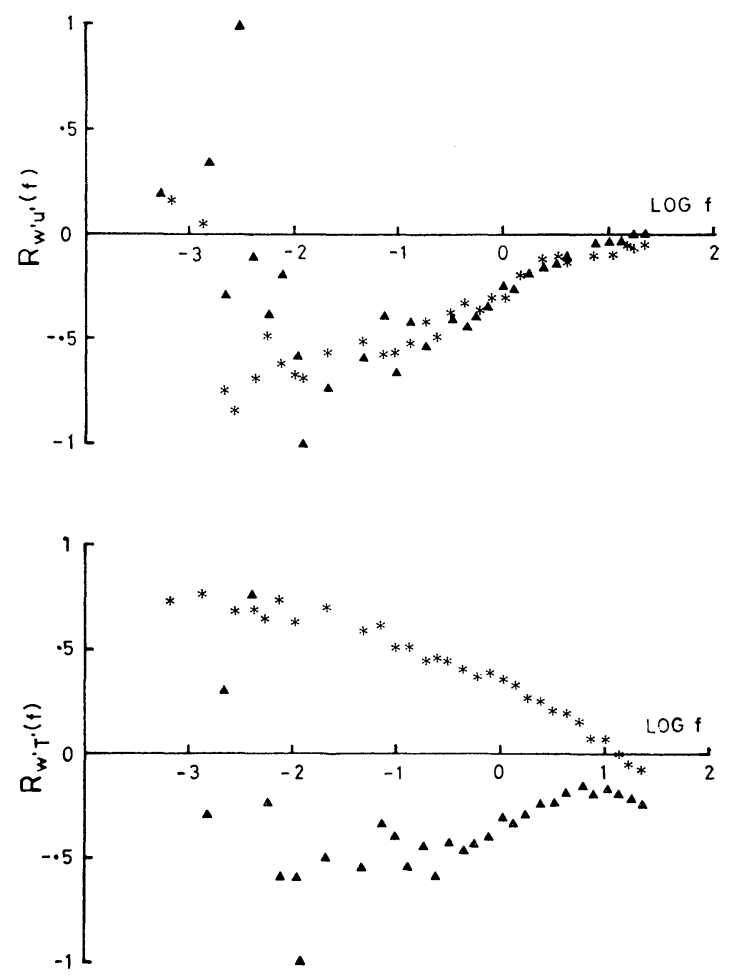

Fig. 1. The frequency correlation of $u^{\prime}-w^{\prime}$ (top) and $T^{\prime}-w^{\prime}$ (bottom) for stable

(A) and unstable $(*)$ cases. 
The joint density functions are obtained on an array of 16 by 16 joint class intervals about the standard signals. Each interval is 0.5 of the standard deviation. This idea is same that Holland (1968) showed.

The turbulence is more isotropic and homogenious in high frequency region than in low frequency region because of the energy transfer and the pressure effect. In low frequency region convective motion is dominant and the turbulence is nonhomogenious. Fig. 1 shows the frequency correlation for $\overline{u^{\prime} w^{\prime}}$ (top) and $\overline{T^{\prime} w^{\prime}}$ (bottom). The frequency correlation is given by

$$
R_{i j}(f)=C_{i j}(f) / \sqrt{\left(\phi_{i}(f) \phi_{j}(f)\right)}
$$

where $C_{i j}(f)$ is co-spectrum of $i$ and $j$ component and $\psi_{i}(f)$ is the spectrum of $i$ component. The absolute value of the frequency correlation decreases under 0.5 in the high frequency region above $0.1 \mathrm{~Hz}$. The turbulence is more random in high frequency region than in low. By the central limit theorem we can expect that distribution is normal where the correlation is small.

To separate this character a running mean filter is used. The high pass filter (H.P.F.) changes an original signal $x_{i}{ }^{\prime}$ to $\widehat{x_{i}}$ by;

$$
\hat{x}_{i}=x^{\prime}{ }_{i+\frac{1}{2} n}-\frac{1}{n} \sum_{j=i}^{i+n} x_{j}^{\prime}
$$

The low pass filter (L.P.F.) does;

$$
\tilde{x}_{i}=\frac{1}{n} \sum_{j=i}^{j_{+} n} x_{j}^{\prime}
$$

The transfer function for L.P.F. is

$$
G(f)=\frac{\sin ^{2} 2 \pi f n \Delta T}{(2 \pi f n \Delta T)^{2}}
$$

where $\Delta T$ is the averaging time of digitization and $n$ the number of the digital samples over which the running mean is taken. Taking 500 for $n$, the cut-off frequency is about $0.5 \mathrm{~Hz}$. This filter roughly divides the fluctuation into the convective eddy and the inertial process.

\section{Discussion.}

The density function is expanded in the GramCharlier series. The dependency of the coefficients on stability is discussed with the corresponding density function and joint density function to make clear the characters of turbulence. The change of the nondimensionalised standard deviation with stability is shown in Fig. 2, the skewness in Fig. 3 and the kurtosis in Fig. 4.

(Longitudinal velocity fluctuation) In the limit of neutral stability, the standard deviation approaches to individual constant. $\sigma_{u} / u^{*}$ at neutral condition is 2.4-2.5 in this experiment. This value agrees well with other results. The skewness $S_{u}$ stays near zero throughout the stability change. The skewness is a measure of the eddy transfer in the relevant direction of the part of the eddy kinetic energy. If there is a gradient of turbulent kinetic energy, the velocity component in that direction of the gradient will have a skewed density. The gradient of turbulent energy in

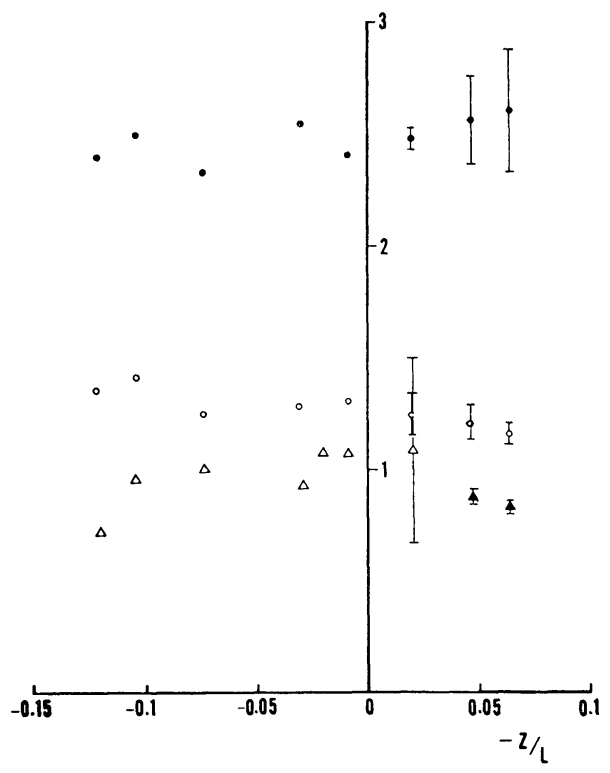

Fig. 2. The variation of the standard deviation with stability; ○, $\sigma_{u} / u^{*} ; \bigcirc, \sigma_{w} / u^{*} ; \triangle, \sigma_{T} / T^{*}$.

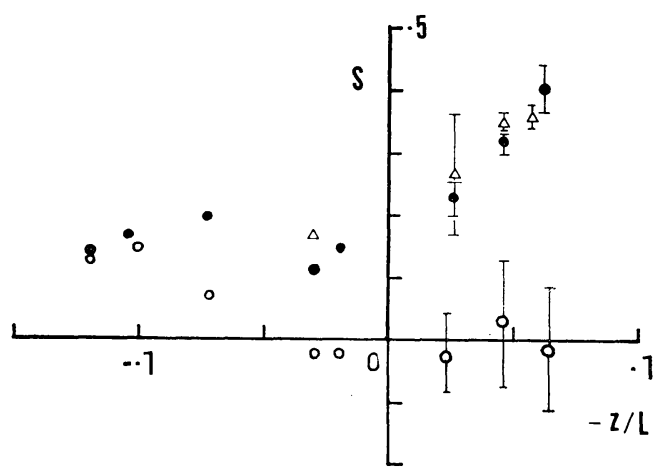

Fig. 3. The variation of the skewness with stability;,$S_{w} ; \bigcirc, S_{u} ; \triangle, S_{T}$. 


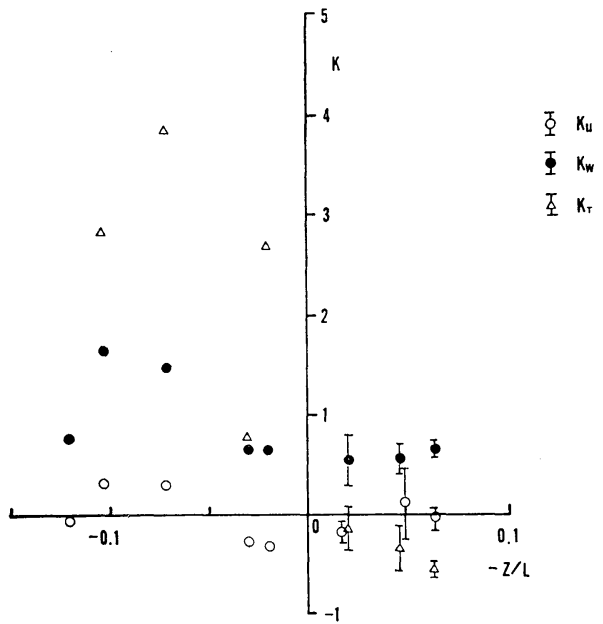

Fig. 4. The variation of the kurtosis with stability;,$K_{w} ; \bigcirc, K_{u} ; \triangle, K_{T}$. horizontal direction is usually smaller than vertical. This is the reason for small $S_{u}$. The kurtosis $K_{u}$ is also smaller than other components and independent from stability like $S_{u}$. This means that the normality is a good approximation for the density function of the longitudinal velocity fluctuation. Fig. 5a shows the normalized density functions, the integral of the density function is unit, of longitudinal velocity fluctuation for stable (top, $z / L=0.074$ ), near neutral (middle, $z / L=$ -0.015 ) and unstable (bottom, $z / L=-0.045$ ). The solid line shows the density function reconstructed by eq. (2) with the corresponding skewness and kurtosis. The broken line shows the normal density function given by eq. (3). The observed density is given by dots. The normal, the reconstructed and the observed density functions do not differ from each other. The normal density function is a good approximation throughout the
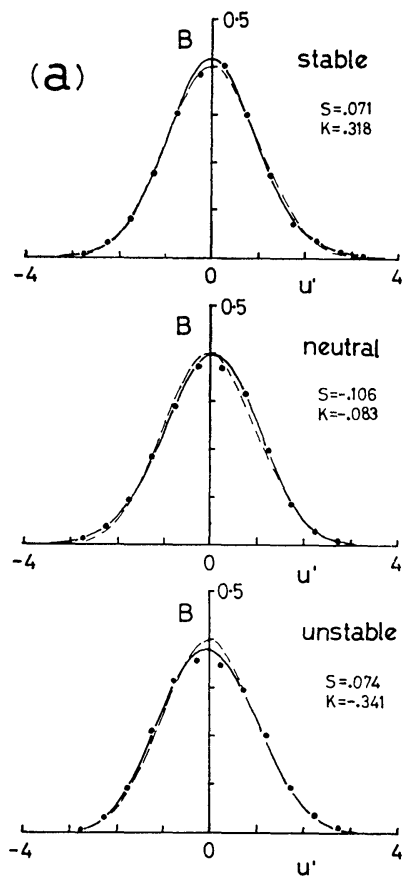
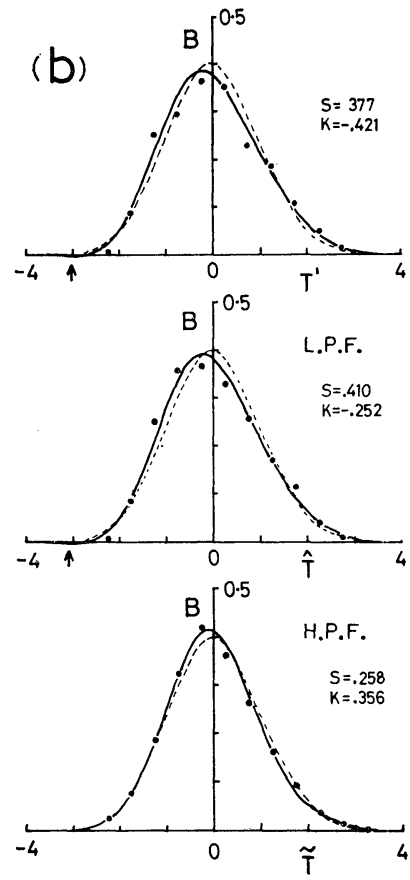
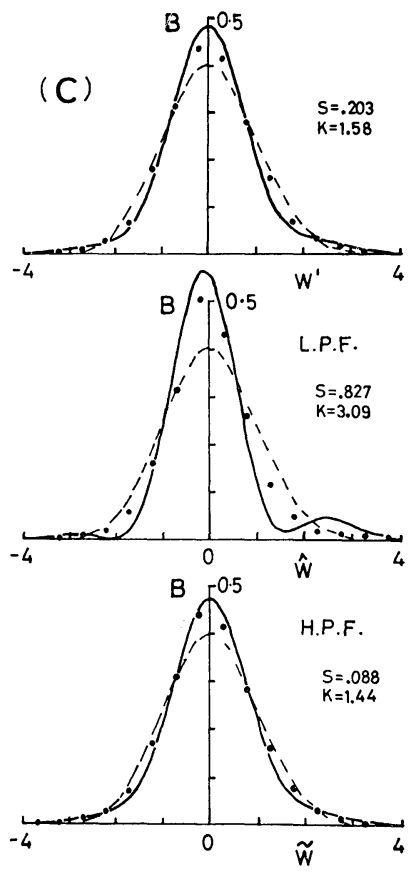

Fig. 5. Normalizes density function. The broken line shows the normal density function, the bold is given by eq. (2) with the corresponding coefficients and dots shows the observed density function.

(a) Of longitudinal velocity fluctuation for each stability category. Stability $z / L$ are 0.074 for stable, -0.015 for near neutral and -0.045 for unstable conditions.

(b) Of vertical fluctuation in stable, without filtering (top), with L.P.F. (middle) and H.P.F. (bottom).

(c) Of temperature fluctuation in unstable, without filtering (top), with L.P.F. (middle) and H.P.F. (bottom). 
stability change.

(Vertical velocity and temperature fluctuation) These components have a similar feature for stability change. The stability dependency of $\sigma_{w} / u^{*}$ and $\sigma_{T} / T^{*}$ shown in Fig. 2 are same as other reports. $S_{w}$ and $S_{T}$ are positive throughout the stability change and increase with unstability. The density function with positive skewness has a maximum density on negative side and spreads on positive side than negative. This feature is exposed clearly in Fig. $5 \mathrm{~b}$ and $5 \mathrm{c}$. Fig. $5 \mathrm{~b}$ shows the density function of vertical velocity fluctuation in stable $(z / L=0.074)$ and Fig. 5c of temperature fluctuation in unstable $(z / L=-0.045)$. The top figure is made by the fluctuation without filtering, the middle with L.P.F. and the bottom with H.P.F. In all cases skewness is positive and the maximum density is on negative side. The tail of the density function spreads wider in positive side, which represents hot or upward air motion, than negative. The corresponding time domain trace could be that the subsiding cold air has longer period and narrower amplitude than the upward hot air.

$K_{w}$ is positive in either stable and unstable. $K_{T}$ is negative in unstable and positive in stable. $K_{w}$ and $K_{T}$ increases with stability. Large kurtosis could correspond to the trace which consists of two periods, period of large amplitude (turbulent) and of small (dissipated) on the same level. The skewness is large in unstable and kurtosis is large in stable. The density function approaches to normal in neutral stability. The skewness and kurtosis in low frequency region are smaller than high frequency in most cases. The density function with H.P.F. is more normal than that with L.P.F. The constructed density function with negative large kurtosis gives an unrealistic negative density function, as shown by arrows in Fig. 5c. Large kurtosis modifies the density function too much as shown in the middle of Fig. 5b. In these cases the density function deviates so much from normal that the Gram-Charlier expansion is not effective; the higher the order of the expansion is the larger the coefficient is. Except such cases, the reconstructed density function represents the observed density function which deviates from normal.

(Correlation coefficient) Two kinds of correlation coefficients of turbulent fluctuation, $\rho_{u w}$ and $\rho_{w T}$, are physical concern. These relate to momentum flux or stress $\tau\left(=\rho u^{* 2}\right)$ and heat flux

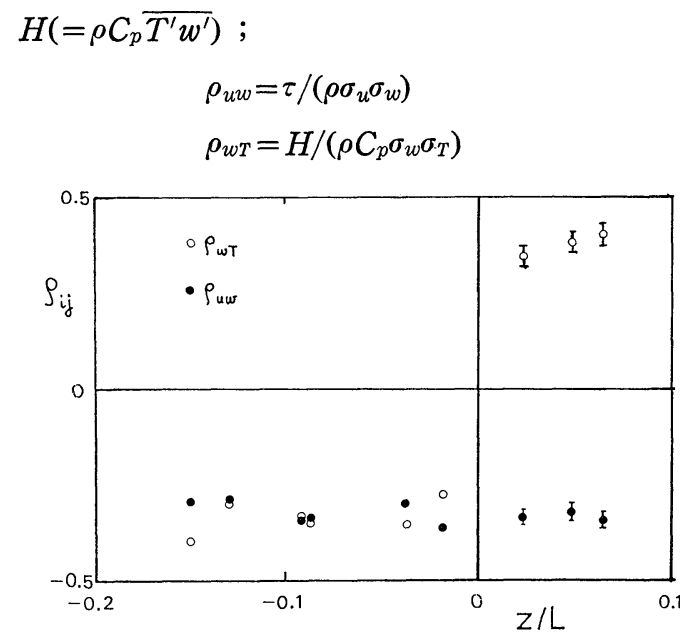

Fig. 6. The variation of the correlation coefficient with stability.

Fig. 6 shows the stability dependency of the correlation coefficients. In surface shear flow $\rho_{u w}$ is always negative and does not seem to depend on stability in this range. The average value is -0.33 . $\rho_{w T}$ changes its sign with stability, positive for unstable and negative for stable. The change is abrupt and $\rho_{w T}$ increases with unstability slightly. The average is 0.37 . This is smaller than the value 0.45 obtained by Telford and Warner (1964) or 0.56 by NIPR group. Both are measured by a air plane in atmospheric
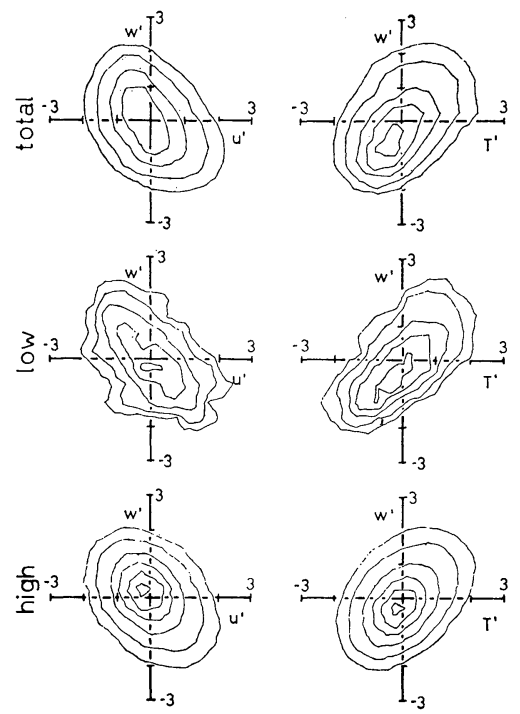

$u^{\prime}-w^{\prime}$

$T^{\prime}-W^{\prime}$

Fig. 7. An example of the joint density function for unstable case, $z / L=-0.045$. 
boundary layer. These large values may associated with large organised convective plume.

(Joint density function) Fig. 7 shows an example of the joint density function for unstable $(z / L=$ -0.045). The left figures show the joint density functions $B\left(u^{\prime}, w^{\prime}\right)$ and the right $B\left(T^{\prime}, w^{\prime}\right)$. The absissas are $w^{\prime}$, and the ordinates $u^{\prime}$ or $T^{\prime}$. The axes run the center of gravity and extend from -3.0 to 3.0 of standard deviation. The top maps are made without filtering, the middle with L.P.F. and the bottom with H.P.F.. The solid lines are contour lines of the observed density which is normalised. Each line shows $0.05,0.1,0.2,0.3$ and so on from out side. The contour lines lean left for negative correlation and right for positive.

In surface shear fiow $w^{\prime}$ correlates with $u^{\prime}$ negatively. The correlation between $w^{\prime}$ and $T^{\prime}$ depends on stability as mentioned before. Comparing the filtered joint density functions the correlations are more significant in low frequency region than in high frequency region. This means that the long period of upward air motion relates to hot and slow period of wind speed. $B\left(T^{\prime}, w^{\prime}\right)$ has a peak in negative $w^{\prime}-T^{\prime}$ region and spreads wider to positive region than negative. In time domain the period of upward and hot air is less frequent and wider in amplitude than the subsiding cold air. The corresponding feature of temperature and vertical fluctuation in low frequency region suggests that the thermal plays an important role for statistical characters in unstable state.

\section{Concluding remarks.}

(1) The density function of the turbulent fluctuation in the atmospheric surface layer is not always normal. The deviation from normal is represented by the expansion coefficients of the Gram-Charlier series. The coefficient, skewness or kurtosis, depends on stability.

(a) $S_{u}$ and $K_{u}$ are independent from stability change and smaller than the other components. $B\left(u^{\prime}\right)$ is normal throughout the stability change.

(b) Skewness is positive throughout the stability change. $S_{w}$ and $S_{T}$ increase with unstability.

(c) The statistics of temperature and vertical velocity fluctuation have similar feature and deviate from normal in either stable and unstable condition.

(d) $K_{w}$ and $K_{T}$ are larger in stable than in unstable. $K_{w}$ is positive in either stability region. $K_{T}$ is positive in stable and negative in unstable.

(e) The normality is a good approximation for the density function in neutral stability.

(2) So far as the deviation from normal is small the Gram-Charlier expansion is effective. The observed density function can be represented by the reconstructed density function with the corresponding coefficients.

(3) On the frequency domain the deviation from normal is significant in low frequency region where the convective eddy is dominant.

(4) $\rho_{u w}$ is constant for stability change; the average is -0.33 . $\rho_{w T}$ increases with unstability.

(5) The joint density function shows those characters mentioned above schematically. It shows the apparent positive correlation between $T^{\prime}$ and $w^{\prime}$ in low frequency region of unstable case; the upward air motion relates to hot and slow period of wind speed.

\section{Acknowledgements}

The author would like to express his gratitude for the guidance given by Prof. Mikio Miyake of the Institute of Oceanography, U.B.C.

\section{References}

Batchelor, G. K., 1953: The Theory of Homogenious Turbulence. Cambridge Univ. Press, 169.

Bendat, J. S. and A. G. Piersol, 1966: Measurement and Analysis of Random data. John Wiley and Sons, 281-282.

Holland, J. Z., 1967: Joint density functions of turbulent variables in the atmospheric boundary layer. Phys, Fluids. Supplement S220-S222.

, 1968: An application of some statistical techniques to the study of eddy structure. USAEC TID-24585.

Kendall, M. G. and A. Stuart, 1969: The Advanced Theory of Statistics. Charles-Griffin, 155-157.

Lumley, J. L. and H. A. Panofsky, 1964: The structure of Atmospheric Turbulence. Interscience Pub., 119-160.

Lumley, J. L., 1970: Stochastic Tools in Turbulence. Academic Press, 34-41.

Priestley, C. H. B., 1959: Turbulent Transfer in the Lower Atmosphere, The Univ. of Chicago Press, 67-71.

Telford, J. W. and J. Warner, 1964: Fluxes of heat and vapor in the lower atmosphere derived from air craft observation. J. Atmos. Sci., 21, 539-548.

Tsvang, L. R. and M. Miyake, 1970: International field experiment in USSR, Bull. Amer. Meteor. Soc., 51, 1150. 


\title{
大気乱流の統計的性質
}

\author{
林 正 康* \\ ブリティシュ・コロンビア大学海洋研究所
}

大気境界層中の乱れの統計量を, 安定度と周波数の領域に扔いて実験的に調べた. 風速の流れ方向と鉛直方向成分 と温度のゆらぎの密度函数を Gram-Charlier 級数に展開し, 係数と乱れの性質とについて議論した. ゆらぎを, 対 流と慣性の領域とに分離するのに数值フィルターを用いた。

* 現在の所属 : 公害資源研究所 